\title{
Low power proton exchange membrane fuel cell system identification and adaptive control
}

\author{
Yee-Pien Yang a,*, Fu-Cheng Wang ${ }^{a}$, Hsin-Ping Chang ${ }^{\mathrm{b}}$, \\ Ying-Wei Ma ${ }^{a}$, Biing-Jyh Weng ${ }^{b}$ \\ a Department of Mechanical Engineering, National Taiwan University, Taipei, Taiwan \\ ${ }^{\mathrm{b}}$ Chung Shan Institute of Science and Technology (CSIST), Armaments Bureau, M.N.D., Taiwan
}

Received 7 September 2006; received in revised form 2 November 2006; accepted 17 November 2006

Available online 26 December 2006

\begin{abstract}
This paper proposes a systematic method of system identification and control of a proton exchange membrane (PEM) fuel cell. This fuel cell can be used for low-power communication devices involving complex electrochemical reactions of nonlinear and time-varying dynamic properties. From a system point of view, the dynamic model of PEM fuel cell is reduced to a configuration of two inputs, hydrogen and air flow rates, and two outputs, cell voltage and current. The corresponding transfer functions describe linearized subsystem dynamics with finite orders and time-varying parameters, which are expressed as discrete-time auto-regression moving-average with auxiliary input models for system identification by the recursive least square algorithm. In the experiments, a pseudo-random binary sequence of hydrogen or air flow rate is fed to a single fuel cell device to excite its dynamics. By measuring the corresponding output signals, each subsystem transfer function of reduced order is identified, while the unmodeled, higher-order dynamics and disturbances are described by the auxiliary input term. This provides a basis of adaptive control strategy to improve the fuel cell performance in terms of efficiency, as well as transient and steady state specifications. Simulation shows that adaptive controller is robust to the variation of fuel cell system dynamics, and it has proved promising from the experimental results.
\end{abstract}

(C) 2006 Elsevier B.V. All rights reserved.

Keywords: PEM fuel cell; System identification; Adaptive control

\section{Introduction}

Concerns for the shortage of fossil fuels and for environmental protection have increased the interest in fuel cells as a power source over the past decade. Proton exchange membrane fuel cells, also known as polymer electrolyte membrane fuel cells, have the advantages of high power and energy density, long cell and stack life, low operation temperature, fast start-up, low corrosion, and higher efficiency, compared to most batteries and other fuel cell technologies. Fuel cells are subject to various situations of time-varying load, during which the air flow, gas pressure, temperature, humidity, membrane hydration must be controlled over a wide range of operation. A series of operations require prompt measurements of system states by a set of sensors, such as flowmeter, thermocouples, pressure

\footnotetext{
* Corresponding author. Tel.: +886 2 33662682; fax: +886223631755. E-mail address: ypyang@ntu.edu.tw (Y.-P. Yang).
}

transducer, voltmeter, hall sensor, hydrogen detector, etc. These signals are fed back to the microprocessor to calculate proper control actions executed by various actuators, such as air pump, humidifier, solenoid valves, fan motors and safety devices.

The complex, nonlinear dynamics of fuel cell systems are usually approximately described by the principles of electrochemistry, fluid dynamics, thermodynamics and heat transfer; and in terms of physical parameters, material properties and universal constants under various assumptions and constraints. The voltage and current variations respond instantaneously to the faster dynamics of fuel cells, involving reactant pressure and flow rate, while the slow dynamics of temperature regulation and heat dissipation dominate the steady state response. Many researchers have devoted themselves to the steady-state model of PEM fuel cells, describing the relationship between physical variables through Nernst equation, gas diffusion equation, concentration, voltage drop equation, etc. Most recently, an increasing number of researches have focused on dynamic models for describing the transient response of PEM fuel cell 


\begin{tabular}{|c|c|}
\hline \multicolumn{2}{|c|}{ Nomenclature } \\
\hline \\
\hline \multicolumn{2}{|c|}{$\begin{array}{l}a, b, c, d \text { 's parameters } \\
A, B, C \text { parameter matrices }\end{array}$} \\
\hline$G(z)$ & transfer function of plant \\
\hline$H(z)$ & transfer function of controller \\
\hline$I_{\mathrm{c}}$ & fuel cell current output (A) \\
\hline K & estimation gain \\
\hline$K_{i}$ & integral gain \\
\hline & proportional gain \\
\hline$N_{\mathrm{A}}$ & air flow rate $\left(1 \mathrm{~min}^{-1}\right)$ \\
\hline & hydrogen flow rate $\left(1 \mathrm{~min}^{-1}\right)$ \\
\hline$p_{i}$ & desired closed-loop poles \\
\hline$P$ & covariance matrix \\
\hline$q$ & forward shift operator \\
\hline$q^{-1}$ & backward operator \\
\hline$R$ & internal resistance of fuel cell (ohm) \\
\hline$T(z)$ & closed-loop transfer function \\
\hline$u$ & input vector \\
\hline$V_{\mathrm{c}}$ & fuel cell voltage output $(\mathrm{V})$ \\
\hline$w$ & noise vector \\
\hline$y$ & output vector \\
\hline$z$ & $z$-transform operator \\
\hline \multicolumn{2}{|c|}{ Greek letters } \\
\hline$\phi$ & observation vector \\
\hline$\lambda$ & forgetting factor \\
\hline$\theta$ & parameter vector \\
\hline$\tau$ & sampling time \\
\hline
\end{tabular}

systems. Pukrushpan et al. [1] described transient dynamics by a set of first order equations governing air compressor, mass transportation, energy conservation of the reactant flows and pressures in the cathode and anode. Ceraolo et al. [2] provided more precise partial differential equations to describe both static and dynamic behaviors of the PEM fuel cell, including gas diffusion, proton concentration, mass transportation, etc. In their spatial, time-dependent fuel cell model, Golbert and Lewin [3] included dynamics of water condensation, evaporation and generation, as well as quasi-steady-state temperature profile. Pathapati et al. [4] derived a dynamic model with the effects of charge double-layer capacitance, dynamics of flow and pressure, and mass/heat transient features of fuel cells, which predicted the transient response of cell voltage, temperature, gas low rates, pressures under sudden change in load current.

No matter how complex the fuel cell dynamic model is, it never describes system responses precisely in terms of system parameters. Their nonlinearity and time varying characteristics inevitably pose difficult problems on system identification and control. Simplified models, either linear or nonlinear, with nominal parameters are usually valid within a linear range of operation. Such models are usually used to investigate stability, sensitivity, observability, and controllability, before designing a controller. More practically, these parameters are varying with time and operating state, and are identified in real time to match system response with minimal errors. Therefore, adaptive control can serve as a feedback law to achieve control objectives subject to the variation of system parameters as well as external disturbances. Pukrushpan et al. [1] designed an observer-based feedback controller to protect the fuel cell stack from oxygen starvation during changes of current command, using the linear quadratic technique based on the linearized state-space model. Paradkar et al. [5] integrated a linearized FEM fuel cell model into a power plant, and simulated a load frequency control problem by an optimal controller based on disturbance accommodation control theory. Schumacher et al. [6] employed a fuzzy controller for the humidity management by adjusting the fan voltage for the air supply to a miniature PEM. Jurado and Saenz [7] addressed that their proposed adaptive control strategy was able to stabilize a hybrid fuel cell and turbine system subject to system parameter variations and external disturbances. Meyer and Revankar [8] surveyed the control-oriented models and control strategies of PEM fuel cell systems. They indicated that future attempts to develop nonlinear multi-input multi-output systems, as this paper proposes, must be important over the entire operating range in order to seek reduced reactant usage under various power demands.

\section{Fuel cell system modeling}

In the papers mentioned above, the PEM fuel cell is basically assembled from a series of cells, each of which is composed of a proton exchange electrolyte sandwiched by anode and cathode electrodes, and each electrode consists of a catalyst and a gas diffusion layer. In order to develop the control strategy of a fuel cell, its dynamic model from previous studies is adopted, describing Nernst equation, cathode gas diffusion, cathode kinetics, and proton concentration dynamics [2,9-11].

From the system viewpoint, hydrogen at a certain pressure is fed at an adjustable flow rate $N_{\mathrm{H}}$, which is regarded as an input variable. On the other hand, air at standard atmosphere of 1 bar is often transported by air pump to provide oxygen to the cathode. The partial pressure of oxygen is proportional to the air pressure, as well as to the flow rate of air $N_{\mathrm{A}}$, which is also considered an input variable of the fuel cell. The cell voltage and current are normally chosen as system outputs. From modern control theories [12], the control-oriented fuel cell system block diagram can be approximated as a two-input two-output system as shown in Fig. 1, and is reduced to a standard diagram with four blocks in Fig. 2. These coupled blocks, denoted by $G_{i}, i=1$, $2,3,4$, describe the relationships between outputs, $I_{\mathrm{c}}$ and $V_{\mathrm{c}}$, and inputs, $N_{\mathrm{A}}$ and $N_{\mathrm{H}}$, where $R$ denotes the internal resistance of the fuel cell. Assuming that the fuel cell is operated at a certain operating point, the four blocks can be linearized as transfer functions of finite orders and time-varying coefficients. More specifically, $G_{1}$ is the transfer function between $I_{\mathrm{c}}$ and $N_{\mathrm{A}}, G_{2}$ between $V_{\mathrm{c}}$ and $N_{\mathrm{A}}, G_{3}$ between $I_{\mathrm{c}}$ and $N_{\mathrm{H}}$, and $G_{4}$ between $V_{\mathrm{c}}$ and $N_{\mathrm{H}}$. Therefore, the fuel cell stack model can be expresses as

$$
\begin{aligned}
& V_{\mathrm{C}}=G_{2} N_{\mathrm{A}}+G_{4} N_{\mathrm{H}}+R I_{\mathrm{C}} \\
& I_{\mathrm{C}}=G_{1} N_{\mathrm{A}}+G_{3} N_{\mathrm{H}}
\end{aligned}
$$




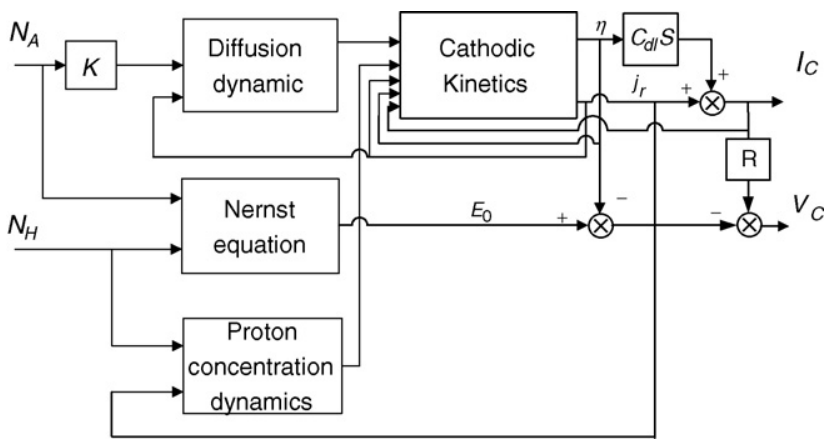

Fig. 1. PEM Fuel cell block diagram.

This configuration is usually employed as the basis of system identification, as well as an adjustable model for designing the fuel cell controller.

\section{System identification}

\subsection{ARMAX model and PE signal}

The linear system model (1) and (2) can be represented as a discrete-time equation of difference operator, and leads to the following autoregressive moving-average with auxiliary input model, the ARMAX model [13]:

$$
\begin{aligned}
& A(q, k) y(k)=B(q, k) u(k)+C(q, k) w(k) \\
& A(q, k)=a_{0}(k)+a_{1}(k) q^{-1}+a_{2}(k) q^{-2}+\cdots+a_{r}(k) q^{-r}
\end{aligned}
$$

$$
\begin{aligned}
& B(q, k)=b_{1}(k) q^{-1}+b_{2}(k) q^{-2}+\cdots+b_{r}(k) q^{-r} \\
& C(q, k)=c_{0}(k)+c_{1}(k) q^{-1}+c_{2}(k) q^{-2}+\cdots+c_{r}(k) q^{-r}
\end{aligned}
$$

where $y$ denotes the system $m$-dimensional output vector, $u$ represents the system $n$-dimensional input vector, $w$ is composed of higher-order dynamics and disturbances, and is known as an estimation error vector. The integer $k$ denotes the time instant

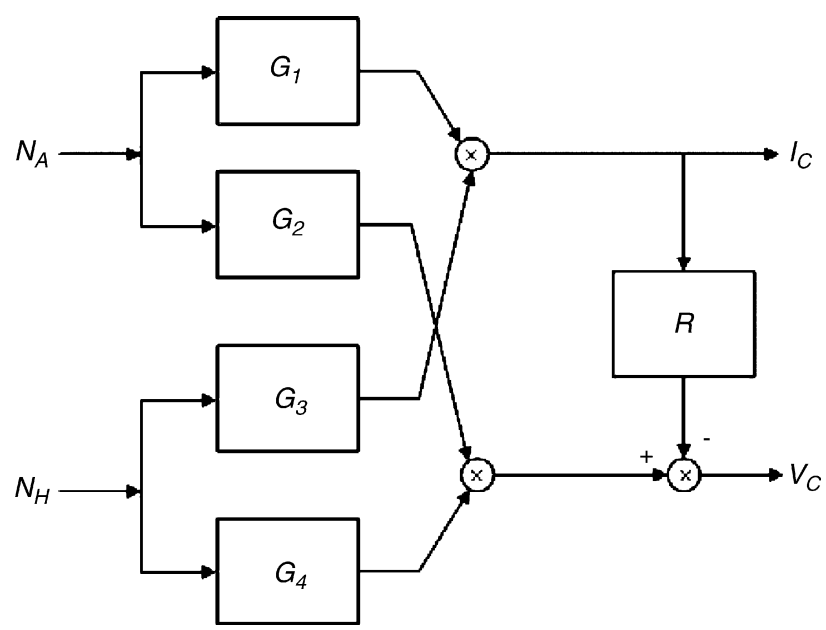

Fig. 2. Approximated two-input and two-output block diagram. of sampling, and $r$ is the system order. Without loss of generality, $a_{0}=c_{0}=1$. In a succinct form, the term $A(q, k) y(k)$ is the autoregression part, where $q^{-1}$ is a backward shift operator and $q^{-h} y(k)$ represents the past output $y(k-h) ; h=1,2$, ... $r ; B(q, k) u(k)$ denotes the moving average of past inputs, where $q^{-h} u(k)=u(k-h)$; and the auxiliary input $C(q, k) w(k)$ is included to better describe the system unmodeled dynamics, including higher-order dynamics and disturbances. Therefore, the corresponding transfer function between outputs and inputs in the discrete-time form becomes

$G_{i}(z)=\frac{B_{i}(z)}{A_{i}(z)}$

where $A_{i}(z)$ and $B_{i}(z)$ are, respectively, the z-transforms of $A_{i}(q)$ and $B_{i}(q), i=1, \ldots, 4$.

According to linear theory, all the system modes can be excited by an impulse input, whose frequency response is an ideal constant over all frequencies and is also known as white noise. That means the frequency contents of an impulse consist entirely of sinusoidal waves of the same amplitude or strength. In the real world, a signal with a rich content of frequencies can be generated by a pseudo-random binary sequence (PRBS), and this signal satisfies the persistent excitation $(\mathrm{PE})$ requirement for system identification.

\subsection{Recursive least square algorithm}

The approach of recursive least square algorithm for system identification is to predict the system output according to the past information of input and output measurements, as well as the update set of system parameters. From (3)-(6), for $a_{0}=c_{0}=1$, the estimation of $y(k)$ is expressed as

$\hat{y}(k)=\phi^{\mathrm{T}}(k-1) \hat{\theta}(k-1)$

where

$$
\begin{aligned}
\phi^{\mathrm{T}}(k-1)= & {[-y(k-1) \cdots-y(k-m) u(k-1)} \\
& \times \cdots u(k-n) w(k-1) \cdots w(k-r)]
\end{aligned}
$$

$\hat{\theta}^{\mathrm{T}}=\left[\hat{a}_{1} \cdots \hat{a}_{r}, \hat{b}_{1} \cdots \hat{b}_{r}, \hat{c}_{1} \cdots \hat{c}_{r}\right]$

and the estimation error $w(k)$ is defined as $y(k)-\hat{y}(k)$.

At step $k$, when the new output is measured, the update set of parameters are calculated by [13]

$$
\begin{aligned}
& \hat{\theta}(k)=\hat{\theta}(k-1)+K(k)\left[y(k)-\phi^{\mathrm{T}}(k-1) \hat{\theta}(k-1)\right] \\
& K(k)=P(k-1) \phi(k-1)\left[\lambda+\phi^{\mathrm{T}}(k-1) P(k-1) \phi(k-1)\right]^{-1}
\end{aligned}
$$

$P(k)=\left\lfloor I-K(k) \phi^{\mathrm{T}}(k-1)\right\rfloor \frac{P(k-1)}{\lambda}$

where the estimation gain $K(k)$ brings the relative information of new measurements to update the parameter estimation, and the covariance matrix $P(k)$ characterizes the difference between the estimated parameters and their true values. Initially, $P(0)$ is 
Table 1

Single fuel cell parameters and specifications

\begin{tabular}{ll}
\hline Rated power & $6 \mathrm{~W}$ \\
Rated voltage & $0.6 \mathrm{~V}$ \\
Rated current & $10 \mathrm{~A}$ \\
Active area per cell & $50 \mathrm{~cm}^{2}(5 \mathrm{~cm} \times 10 \mathrm{~cm})$ \\
Anode reactant & Pure compressed hydrogen, $0.5 \mathrm{SLPM}$, \\
& $6 \mathrm{psig}$ \\
Cathode reactant & Humidified air, $3 \mathrm{SLPM}, 0.05 \mathrm{psig}\left(50^{\circ} \mathrm{C}\right.$, \\
& $90-100 \%$ humidity $)$ \\
Stoichiometry & $\mathrm{H}_{2}: 1.5$, Air: 3 \\
Operating temperature & $295 \mathrm{~K}\left(22^{\circ} \mathrm{C}\right)$ \\
Ambient temperature & $293 \mathrm{~K}\left(20^{\circ} \mathrm{C}\right)$ \\
Current density & $0.2 \mathrm{~A} \mathrm{~cm}-2$ \\
Cell voltage & $0.63 \mathrm{~V}$ \\
\hline
\end{tabular}

chosen a large positive number, for example $10^{3} I$, where $I$ is an identity matrix, to render the inaccurate initial guess $\hat{\theta}(0)$ quickly negligible. The coefficient $0<\lambda<1$ is called the forgetting factor, which places more importance on the new information for updating system parameters, and less attention on the past information.

\subsection{Experimental setup}

The system identification is performed on a single cell of a square active area of proton exchange membrane. The system parameters and rated operating specifications are given in Table 1. The flow rate of humidified air is supplied by an air pump, while the flow rate of hydrogen is regulated by a solenoid valve. Either output cell voltage or current can be tuned at a specific value with an electronic load meter. Each transfer function $G_{i}$ is identified by measuring the corresponding set of input and output, while fixing the other set of input and output at their nominal values. For example, $G_{1}$ is the transfer function between cell current and air flow rate, while cell voltage and hydrogen flow rate are fixed; $G_{4}$ is the transfer function between cell voltage and hydrogen flow rate, while cell current and air flow rate are fixed.

The excitation PRBS signal between 2 and 0.5 standard liter per minute (SLPM) is used for all experiments. This PRBS signal that resembles pure white noise is easily realized from a combination of a series of square waves of random time duration, whose spectrum contains a rich content of sinusoidal wave and is enough to excite all the system dynamics of fuel cell. The system response to the PRBS is then expected to be a combination of step responses, and the input-output information is used to construct the transfer function of fuel cell at a certain operating point. The input and output data are sampled at $1 \mathrm{~Hz}$, and are manipulated by the MATLAB solver armax to calculate coefficients of transfer functions $G_{i}$. From the step response characteristic shown in Fig. 3, the order of 2 is chosen for the transfer function in the following process of identification. The identified transfer functions are summarized in Table 2 , and their corresponding frequency responses are illustrated in Figs. 4 and 5.

These second-order models of the PEM fuel cell provide significant information as to system dynamical properties. First, the
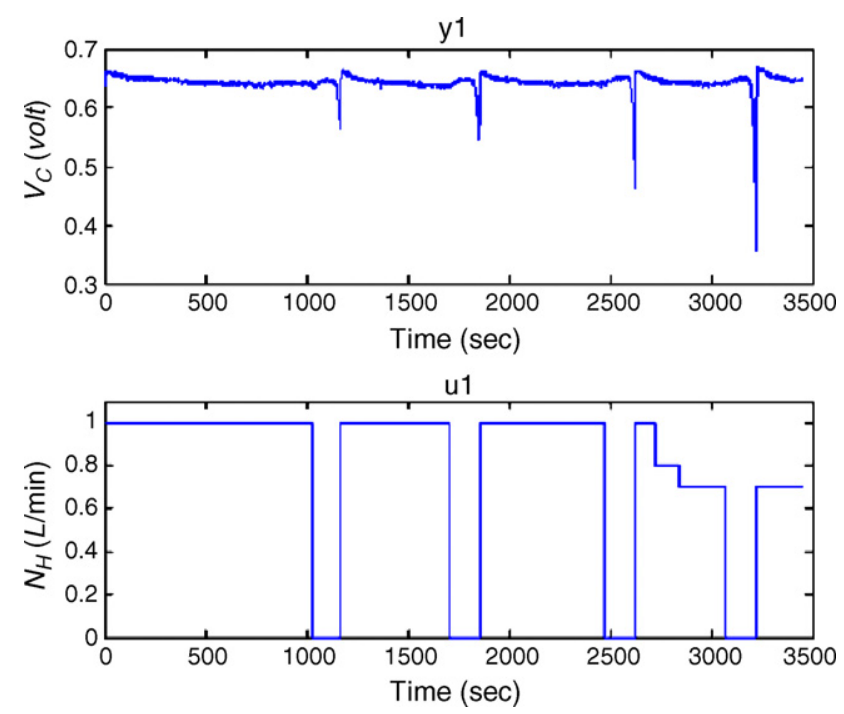

Fig. 3. Hydrogen flow rate vs. fuel cell voltage.

complexity of the fuel cell system can be reduced to a two-input two-output configuration. Although this configuration may not represent system dynamics for the whole range of operation, it provides a basis for the following design of adaptive or robust controller. Second, the set of constant parameters in the second order transfer function is an effective description of system dynamics for a short time span. This is justification for the reason of linearization of a nonlinear system around a specific operating point. Third, the bandwidth of each subsystem indicates how fast the system output responds to various inputs. Therefore, engineers understand how the rate of change of hydrogen and air supply is moderate, and how the controller is designed to improve the closed-loop performance.

\subsection{System identification with higher orders}

To investigate the adequacy of the order of transfer functions, higher order models are also identified and compared with the second order models. For example, in Fig. 6, subsystem $G_{4}$ between cell voltage and hydrogen flow rate shows the Bode plot of identified transfer functions of order 2, 4 and 6 . These models do not have significant differences except for higher frequencies, so a second-order transfer function is sufficient to describe the fundamental dynamics of the PEM fuel cell system.

Table 2

Identified transfer functions

\begin{tabular}{ll}
\hline Transfer function & Operation conditions \\
\hline$G_{1}=\frac{6.6 \times 10^{-3}(z-0.58)}{z^{2}-1.31 z+0.31}$ & $N_{\mathrm{H}}=0.5 \mathrm{SLPM}, V_{\mathrm{c}}=0.6 \mathrm{~V}$ \\
$G_{2}=\frac{2.2 \times 10^{-4}(-0.85)}{z^{2}-1.36 z+0.36}$ & $N_{\mathrm{H}}=0.5 \mathrm{SLPM}, I_{\mathrm{c}}=10 \mathrm{~A}$ \\
$G_{3}=\frac{6.2 \times 10^{-2}(z-1.08)}{z^{2}-1.42 z+0.43}$ & $N_{\mathrm{A}}=3$ SLPM, $V_{\mathrm{c}}=0.6 \mathrm{~V}$ \\
$G_{4}=\frac{6.6 \times 10^{-4}(z-1.12)}{z^{2}-1.38 z+0.38}$ & $N_{\mathrm{A}}=3$ SLPM, $I_{\mathrm{c}}=10 \mathrm{~A}$ \\
\hline
\end{tabular}



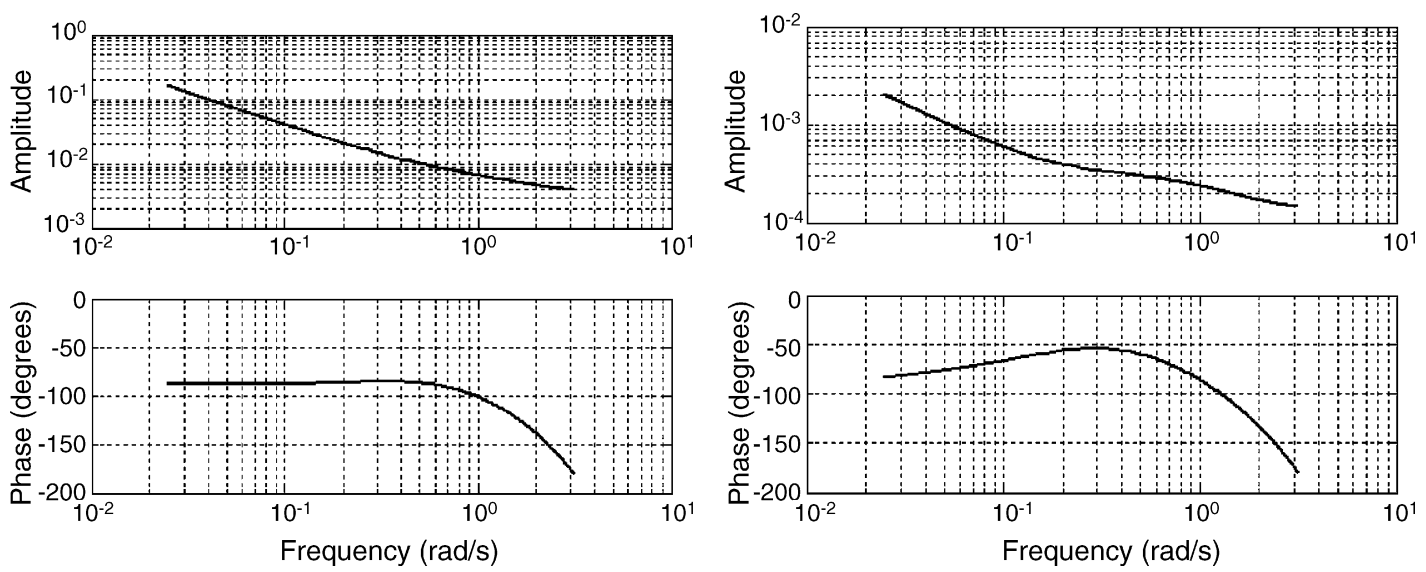

Fig. 4. Frequency response of subsystem $G_{1}$ and $G_{2}$ (2nd order).
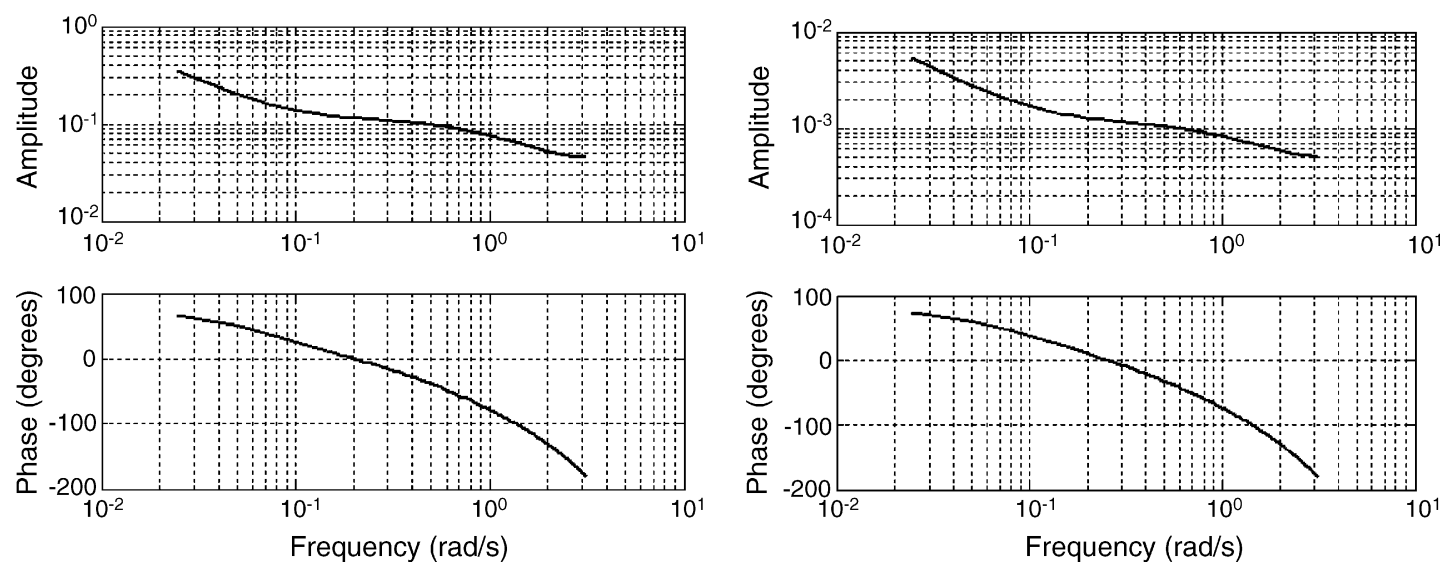

Fig. 5. Frequency response of subsystem $G_{3}$ and $G_{4}$ (2nd order).

\section{System model with time-varying parameters}

In the previous sections, the nonlinear, time-varying fuel cell system was operated over $450 \mathrm{~s}$, and yields a set of convergent parameters that are used to describe system dynamics in the steady state. Note that the random on-off operation of a fuel cell system is not suitable for normal operation, and this procedure is only realized for the purpose of system identification. It is also
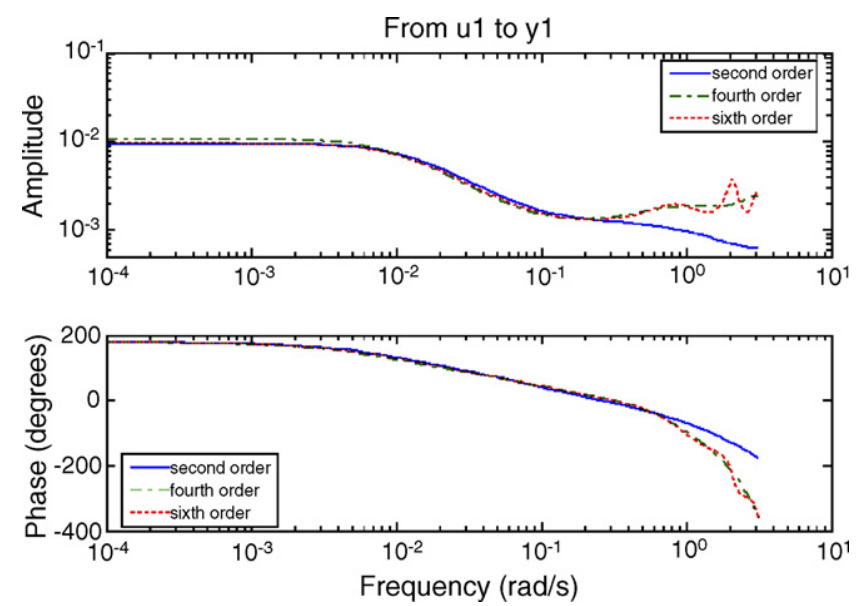

Fig. 6. Bode plots of subsystem $G_{4}$ of different orders. not reasonable to express a nonlinear, time-varying system with a set of transfer functions of finite orders and constant parameters. This is accounted and ensured by fitting the experimental response with the identified time-invariant transfer functions as shown in Fig. 7. The responses they do not fit well give strong evidence that the time-invariant transfer functions are not able to describe true system dynamics.

Therefore, it is reasonable to investigate the history of parameter variations in Fig. 8 during the identification process. It is not surprising that all parameters vary with time to reflect the nonlinear and time-varying properties of fuel cell systems. The corresponding responses simulated by the second-order transfer functions with time-varying parameters fit closely with those from experiments, as shown in Fig. 9. This evidence of excellent response curve fitting with time-varying parameters provides a strong basis for the application of adaptive control to improve fuel cell system performance.

\section{Adaptive controller design}

To introduce the adaptive control and examine its efficacy, the control strategy is simplified to regulate output voltage by adjusting air flow rate subject to plant variations, while the load current and hydrogen flow rate remain constant. In other words, the fuel cell dynamics are simplified as a single-input single out- 

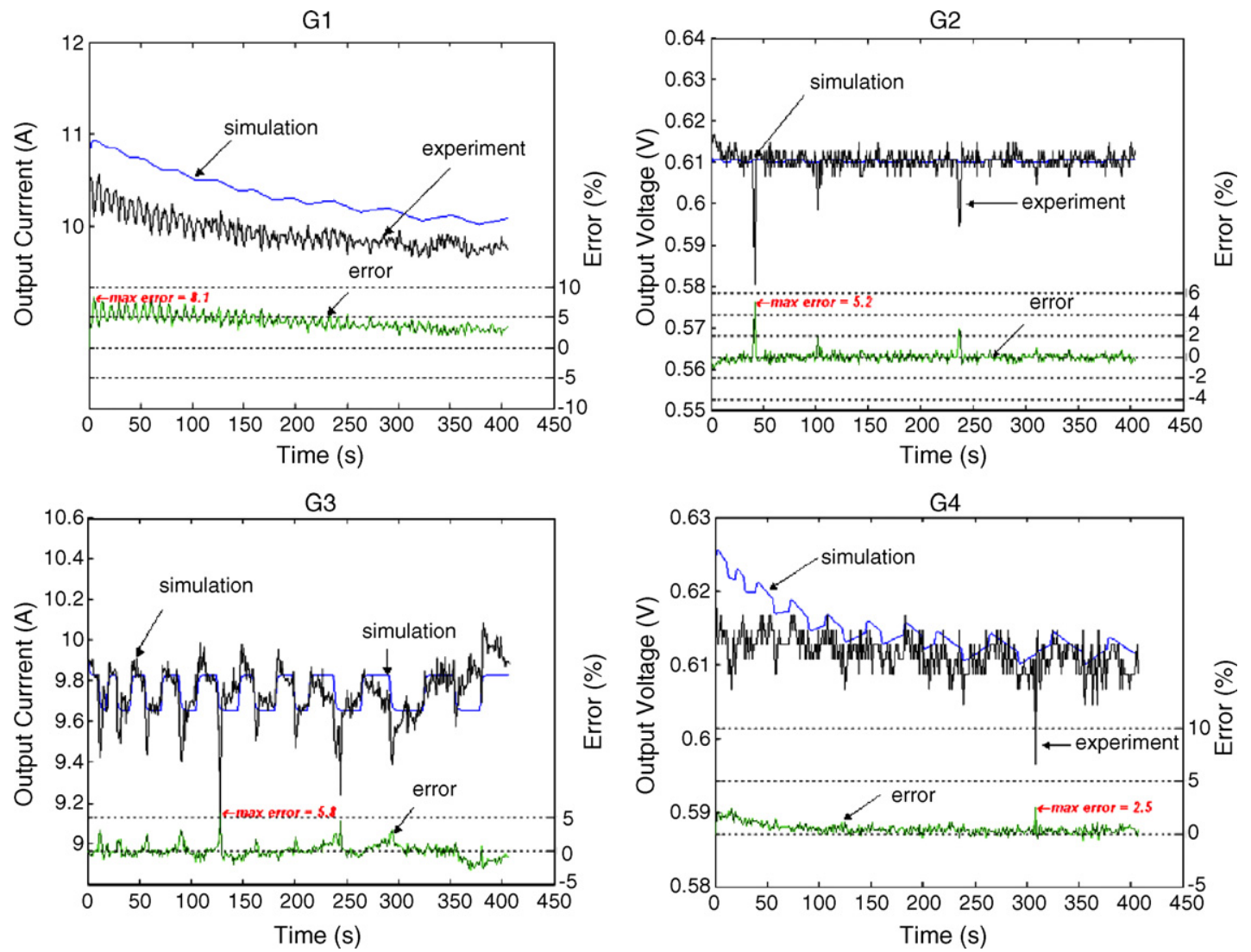

Fig. 7. Experimental response verse simulative response with transfer functions of constant parameters (upper: $G_{1}$ and $G_{2}$, lower: $G_{3}$ and $G_{4}$ ).
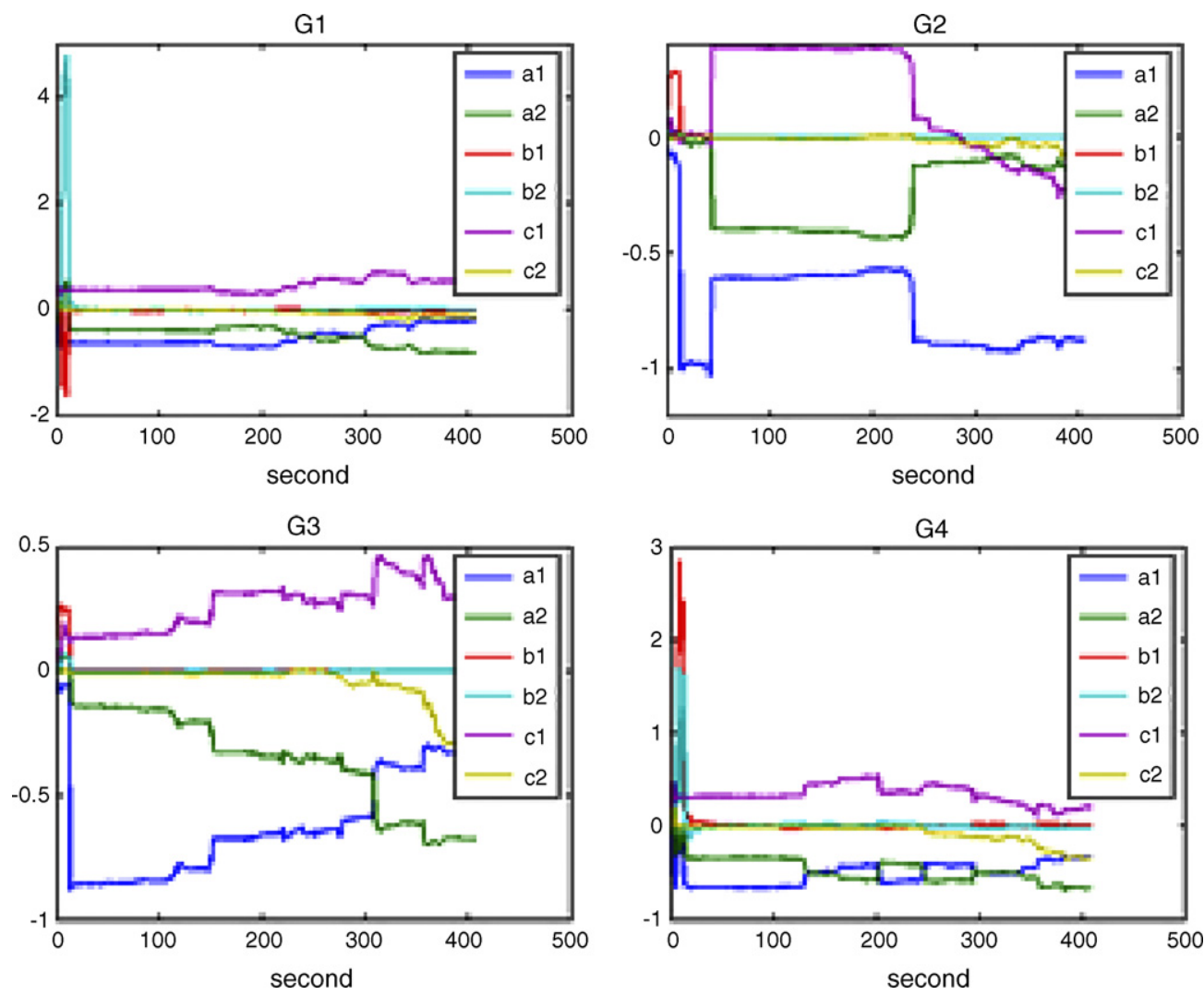

Fig. 8. Variation of system parameters (upper: $G_{1}$ and $G_{2}$; lower: $G_{3}$ and $G_{4}$ ). 

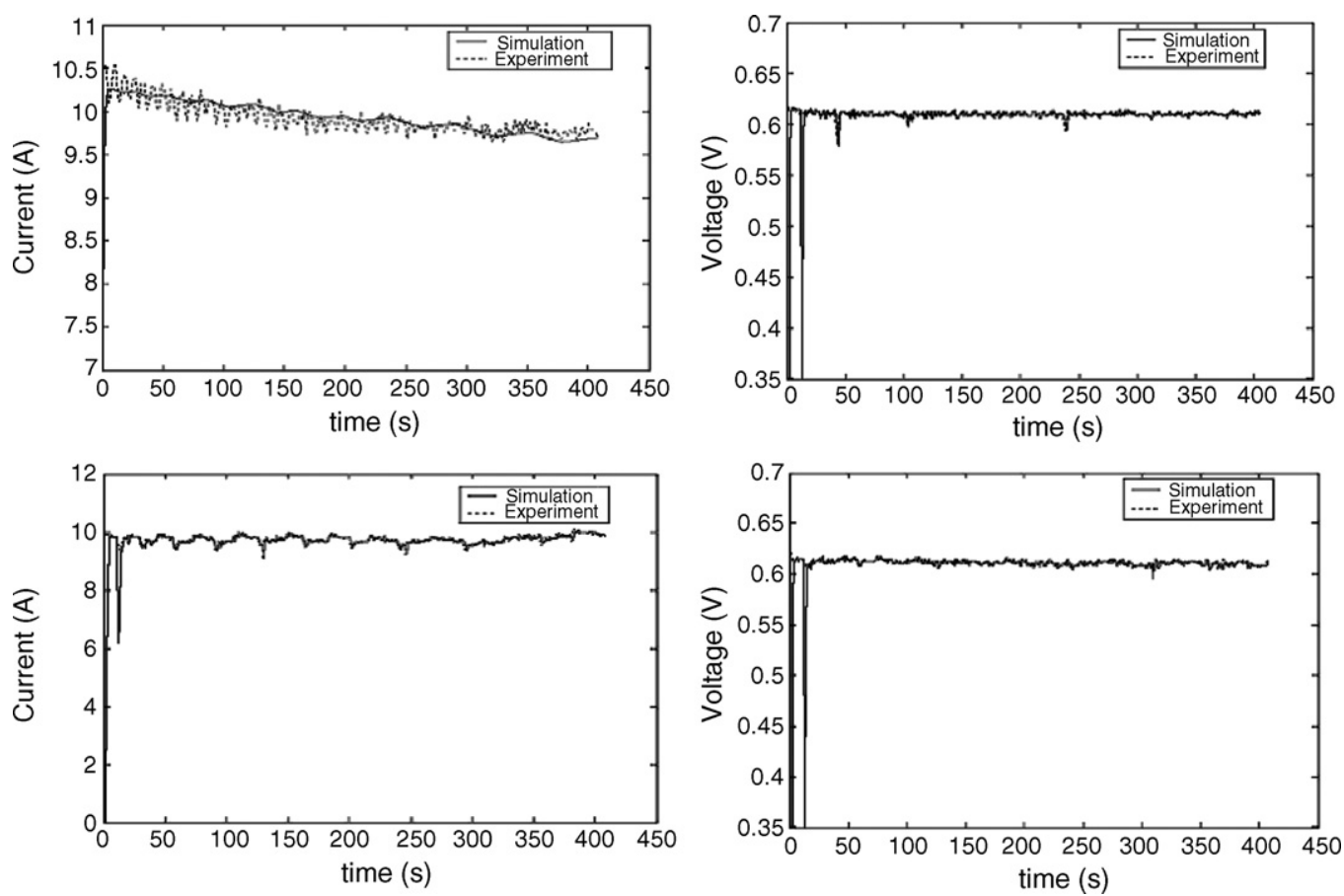

Fig. 9. Response fitting between experiments and simulations by transfer functions with time-varying parameters.

put model $G_{2}$ to describe the relationship between cell voltage and air flow rate. The reason why the $G_{2}$ is chosen for simulation is to compare the experimental results in the following section, where a non-commercialized prototype from a collaborating research group is used and its inlet flow rate of hydrogen is not adaptively controlled but adjusted by a solenoid valve in a scheduled sequence. Moreover, the output current of fuel cell is usually requested by demand and is regarded as an external disturbance. Therefore, the control of cell voltage by the air flow rate is significantly investigated in this paper, as a foundation of exploring multi-variable controls.

The adaptive control strategy includes the specification of desired closed-loop poles, on-line parameter identification and calculation of control gains, so that the output is regulated at a desired command. This adaptive control scenario is illustrated in Fig. 10 and is demonstrated in the following simulations. The desired closed-loop poles are chosen to satisfy the requirements of stability and performance so that the fuel cell output voltage is regulated at a desired level under load and system variations. These variations are detected by the on-line parameter identifier from which new parameters are estimated to update new control actions.

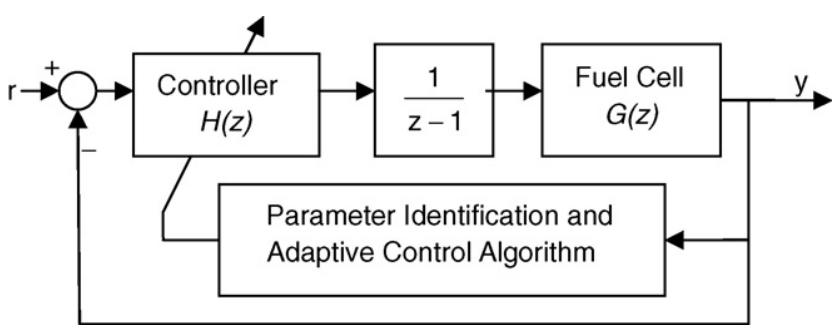

Fig. 10. Adaptive control scenario of fuel cell.
From previous results of system identification, it is sufficient to approximate $G_{2}$ as a second-order transfer function,

$G(z)=\frac{b_{0} z+b_{1}}{z^{2}+a_{1} z+a_{2}}$

An integrator is added so that the plant is augmented to be a thirdorder system; it has memory to keep the past information and enables the controller to accumulate enough effort to eliminate errors in the steady-state [12]. When the feedback controller is chosen as a second-order model

$H(z)=\frac{c_{2} z^{2}+c_{1} z+c_{0}}{z^{2}+d_{1} z+d_{2}}$

the corresponding closed-loop transfer function becomes

$T(z)=\frac{G(z) H(z) /(z-1)}{1+G(z) H(z) /(z-1)}$

Its characteristic equation becomes

$$
\begin{aligned}
& (z-1)\left(z^{2}+d_{1} z+d_{2}\right)\left(z^{2}+a_{1} z+a_{2}\right)+\left(b_{0} z+b_{1}\right) \\
& \quad \times\left(c_{2} z^{2}+c_{1} z+c_{0}\right)=\left(z-p_{1}\right)\left(z-p_{2}\right)\left(z-p_{3}\right) \\
& \quad \times\left(z-p_{4}\right)\left(z-p_{5}\right)
\end{aligned}
$$

where the $p_{i}$ 's are desired closed-loop poles placed inside the unit circle, which is the stable region of poles for a linear discretetime system. Therefore, the control parameters $c$ 's and $d$ 's can be calculated by the desired closed-loop poles and update system parameters at each sampling instant. The simulation is performed by Simulink as shown in Fig. 11. The plant is a two-input two-output transfer function matrix of four elements, in which each element represents a subsystem expressed as a higher-order (4th or 6th order) transfer function, which has been identified in 


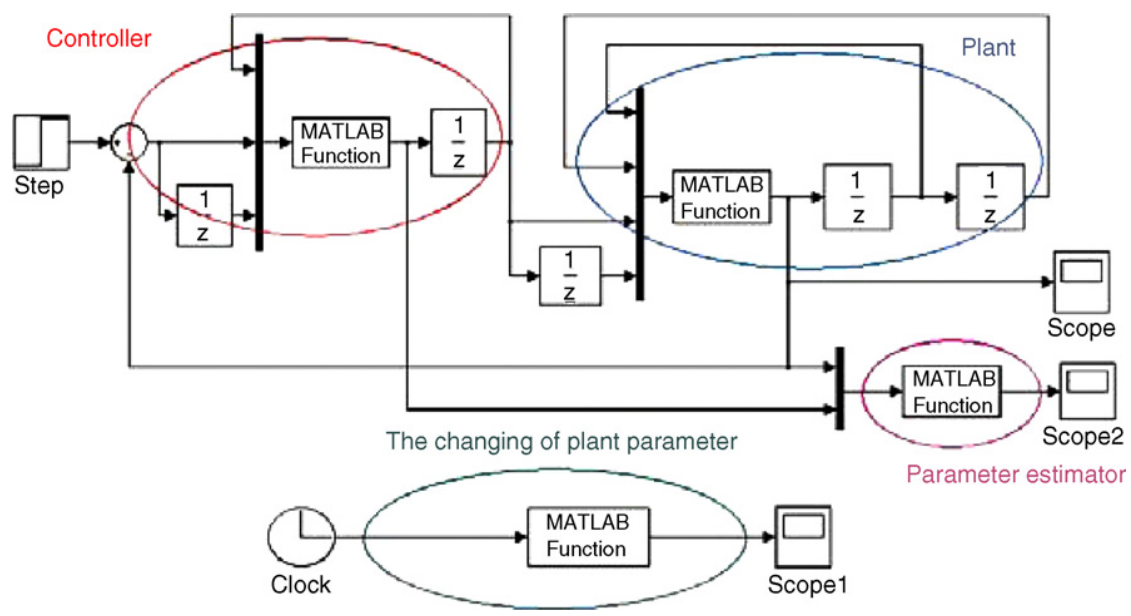

Fig. 11. Adaptive control simulation by Simulink.

the previous sections. The auxiliary input term is also included to introduce higher-order dynamics and external disturbances, so that it is more realistic to simulate a real PEM fuel cell system. The system identification is concurrently processed to obtain a set of system parameters of a reduced second-order system model (14).

During the system identification, the variable forgetting factor is applied to accommodate current status of time-varying system dynamics [13]:

$\lambda(k)=\lambda_{0} \lambda(k-1)+\left(1-\lambda_{0}\right)$

where the initial forgetting factor is $\lambda(0)=0.95$ and $\lambda_{0}=0.99$.

In the simulation, the load current and hydrogen flow rate are both fixed, while subsystem $G_{2}$ is simplified as a second-order transfer function with parameters varying at 150, 200, 250,300, 400 , and $450 \mathrm{~s}$, as shown in Fig. 12. The sampling time is $0.005 \mathrm{~s}$, and the estimated parameters are given in Fig. 13 with minor differences. The corresponding output voltage changes abruptly as the plant varies, but the adaptive controller soon obtains the correct information and adjusts the input air flow rate to achieve the desired command as shown in Fig. 14. The zoom-in part clearly illustrates the fast adaptation of control toward command but with a slight oscillation that demonstrates an inheritance of second-order systems.

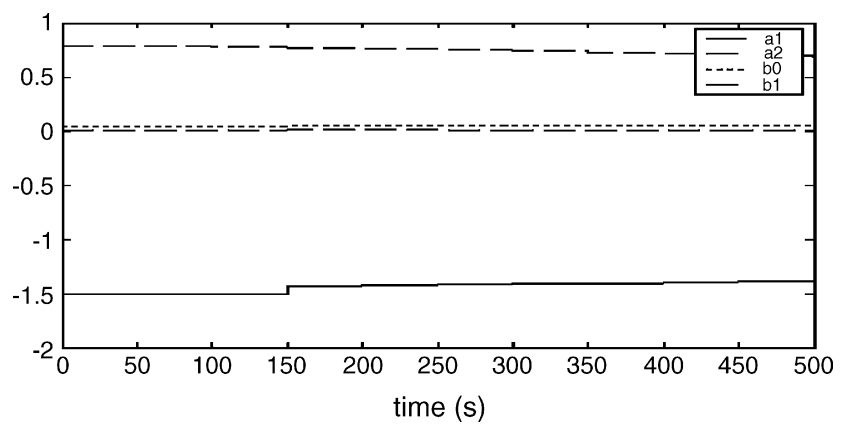

Fig. 12. Parameters of 2 nd order time-varying subsystem $G_{2}$.

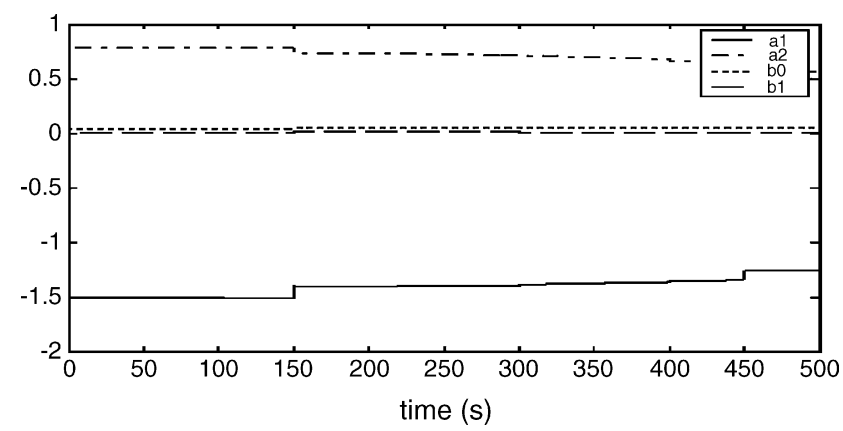

Fig. 13. The estimated parameter of subsystem $G_{2}$.

\section{Experimental results}

The PEM fuel cell for the experiment is a $100 \mathrm{~W}$ stack of $50 \mathrm{~cm}^{2}$ cross-sectional area, with rated voltage and rated current at $10 \mathrm{~V}$ and $10 \mathrm{~A}$, respectively, which is a non-commercialized prototype from a collaborating research group. Therefore, the inlet flow rate of hydrogen is not adaptively controlled but

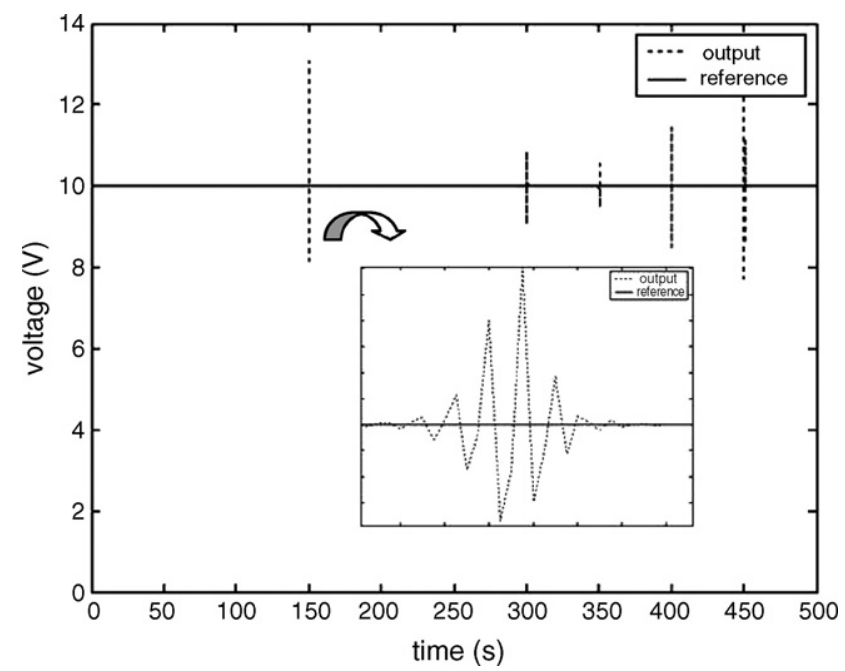

Fig. 14. Adaptive control performance of subsystem $G_{2}$ and zoom-in response at the 150th second. 


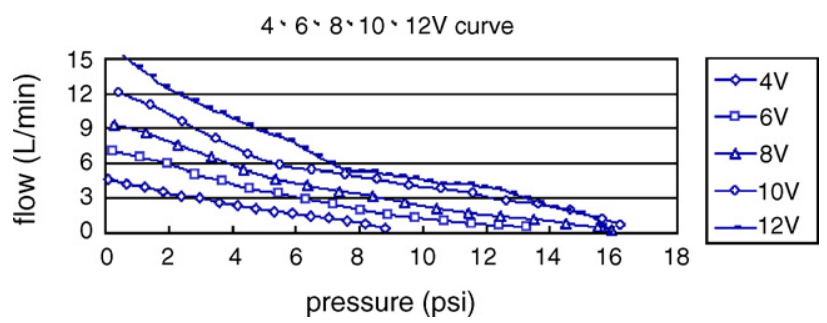

Fig. 15. Air flowrate vs. pressure of air pump.

adjusted by solenoid valve, which is on for $3 \mathrm{~s}$ and off for the other $60 \mathrm{~s}$ at inlet pressure of $6 \mathrm{psi}$, so that a sufficient amount of hydrogen is supplied to maintain a rated operation. The inlet air pressure is also kept at 6 psi by adaptively adjusting the input voltage of air pump according to the flow rate and pressure curve in Fig. 15. That means the adaptive control strategy on the fuel cell stack is examined on the single-input and single output basis. More specifically, the fuel cell output voltage is regulated by adaptively adjusting the voltage of air pump that in turn changes air flow rate, under various request of current from load, while the hydrogen is supplied by a fixed valve with on-off process.

The experimental setup of fuel cell stack and its peripheral devices are schemed in Fig. 16. The on-line parameter identification and adaptive control law are performed with Matlab on a laptop computer, while the fuel cell power is dissipated on an electronic load meter.

Both the plant and controller were modeled as second-order transfer functions in the simulation. To expedite the process of on-line parameter identification and adaptive control, a firstorder plant and PI-controller are chosen for simplicity, and expressed as

$G(z)=\frac{b}{z+a}$

and

$H(z)=K_{\mathrm{p}}+K_{i} \frac{\tau}{z-1}$

The desired closed loop poles are placed at -0.1 and -0.2 inside the unit disk, and the sampling time $\tau$ is chosen at $0.001 \mathrm{~s}$. At each sampling instant, the output voltage and the parameters $a$ and $b$ are estimated in real time. The control gains $K_{\mathrm{p}}$ and $K_{i}$ are calculated during the same sampling period and used at the next sampling instant. Initially, the fuel cell stack is warmed up

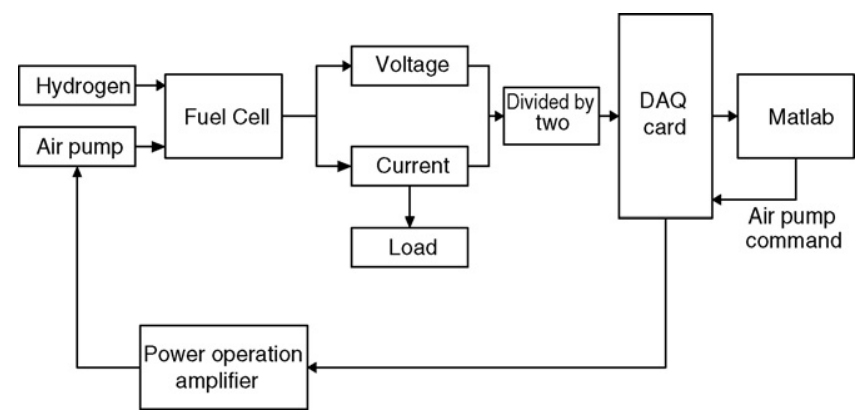

Fig. 16. Block diagram of experimental set-up.

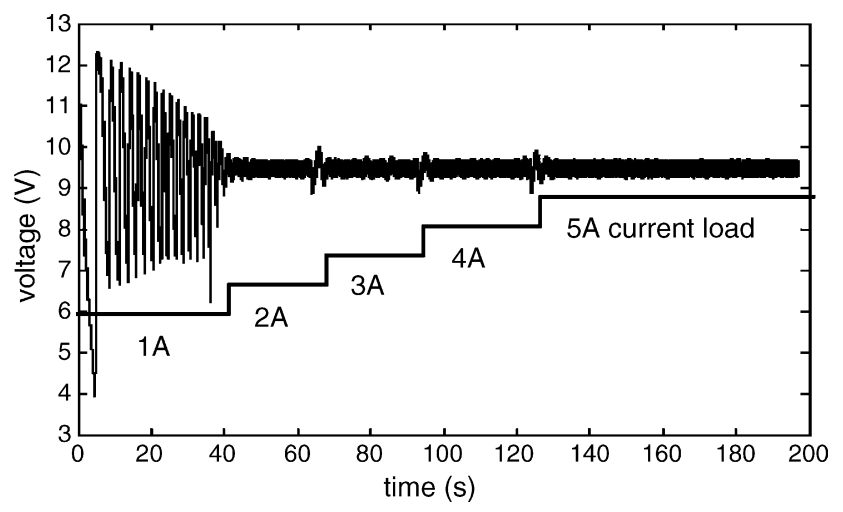

Fig. 17. Adaptive voltage regulation under various current load.
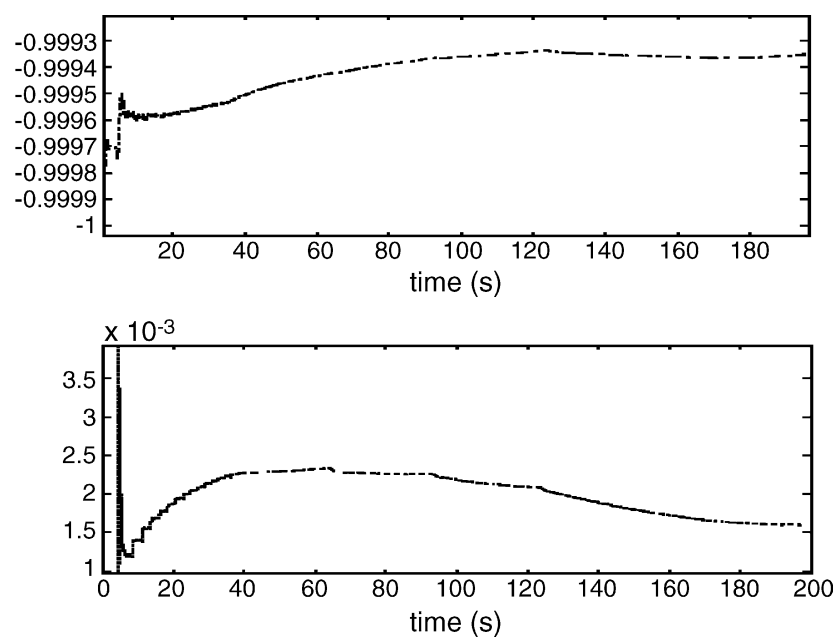

Fig. 18. Variation of parameters $a$ (lower) and $b$ (upper) during adaptive control.

without loading. The desired output voltage is assigned at $9.5 \mathrm{~V}$, while the load increased from 1 through 5A for each 20-30 s. It is quite surprising that the output voltage follows the constant command with minor oscillatory deviation in Fig. 17, even though system parameters $a$ and $b$ are varying as load varies, as shown in Fig. 18. During the adaptation process, the air flow rate is adjusted by the continuous tuning of air pump voltage as displayed in Fig. 19.

In practice, the cell voltage remains unchanged for stationary applications when the air flow is well above stoichiometry, typically twice as much, and the water and thermal manage-

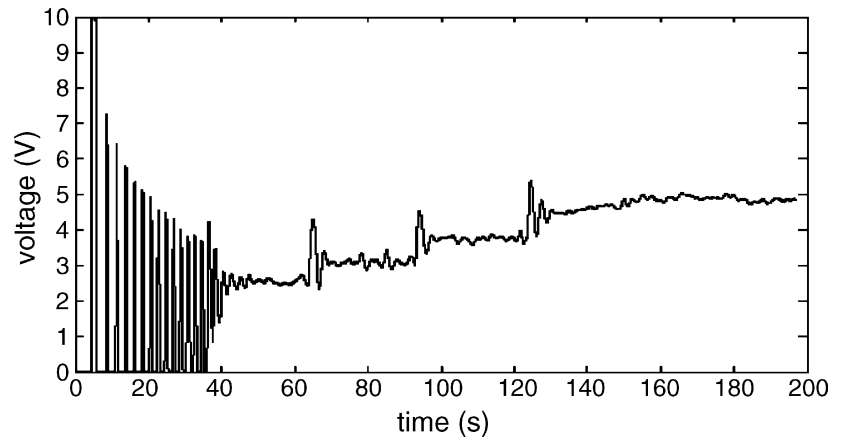

Fig. 19. Input tuning of air pump voltage. 
ment are well controlled [14]. However, the great merit of the adaptive control is to adjust a proper amount of air stoichiometry on a wide range of power demand by changing the air pump speed accordingly, hence excessive power of the air pump can be restrained. It was found that the efficiency was higher when the fuel cell was operated at a fixed stoichiometry by varying the compressor speed than by keeping it constant [15]. Moreover, a fuel cell may not only be sensitive to the air stoichiometry in a restricted range of 1.5 or less, but it is also variable to the operating temperature, humidity, and cell aging.

An obvious question would be why the simplest linear model of first-order is sufficient to describe a nonlinear, time-varying fuel cell system that includes a fuel cell stack, air pump, solenoid valves, and electronic components. From the perspective of $\mathrm{cer}$ tainty equivalent principle in system identification and adaptive control theories, a linear model of reduced order with timevarying parameters is usually used to generate control law as if it were a true system. In other respects, the fuel cell plant is composed of slow dynamics and fast dynamics; the latter decay fast while the former remain as dominant dynamics. For example, the rotational speed of air pump usually presents a faster response than the double layer capacitor property of fuel cell stack, while the chemical reactions in fuel cell display much faster response than the double layer capacitor. Therefore, a lower-order ordinary differential equation is usually sufficient to describe the dominant dynamics of fuel cell system. According to the previous section on system identification, the second-order time-invariant linear model has been proved very reliable to describe fuel cell stack dynamics. In this experiment, the firstorder system model with time-varying parameters becomes a successful candidate to catch the dominant dynamics of a fuel cell stack and its peripheral devices.

The second question is that the adaptive control may not be robust to parameter variations and external disturbances. This can be observed from Fig. 17, where the output voltage is very oscillatory when the requested current is $1 \mathrm{~A}$. This can be explained from two standpoints. First, the hydrogen is consecutively provided in an on-off process of solenoid valve for various current load, so it may have been oversupplied for the low request of current at $1 \mathrm{~A}$. The voltage soon climbs over $9.5 \mathrm{~V}$, and the air pump shuts down immediately due to oversupply of air. The lack of oxygen due to the reduced air supply soon reduces the output voltage, at which point the adaptive controller asks the air pump to start again for more air supply, thereby causing undesirable oscillations.

Second, at the initial stage of system identification, the adaptive control experiences a tremendous change of parameters due to the convergence problem as shown in Fig. 18. This uncertainty in the model causes erroneous calculations on the adaptive control law by an over-simplified system model, thereby perturbing the response from the desired command. This also indicates the lack of robustness of output voltage to the air flow rate. In other words, the fuel cell voltage is very sensitive to the air flow rate. This can be resolved by tuning smaller control gains, or by introducing an optimal control objective which weighs the difference between two successive controls to suppress con- trol oscillations. This also reduces the consumption of parasitic power greatly, thereby increasing the efficiency of the fuel cell system.

It is worth noting that this adaptive scheme may not be the only or independent choice of control for the fuel cell system. A hybrid control perspective for the combination of conventional process or logic control, PID control, optimal control, robust control, stochastic control, H-infinity control, neural network control, fuzzy control, or any advanced control scheme is most likely to succeed in the complex fuel cell system.

\section{Summary and conclusions}

System identification and adaptive control have performed successfully on a low power PEM fuel cell. The highly nonlinear, time-varying, distributed parameter fuel cell system of complex electrochemical reactions has been approximated by a two-input two-output system, described with four system blocks. These blocks describe the dynamic relationship between outputs of cell voltage and current as they relate to inputs of hydrogen and air flow rates in terms of transfer functions that are time-varying as if they were a true system with true parameters. The design of adaptive controller is based on this according to the perspective of certainty equivalence principle. The simulation and experimental results show that the adaptive control is effective for the fuel cell system subject to the change of system parameters and external disturbances. The robustness of the adaptive control strategy may not be guaranteed in terms of model uncertainty, parameter perturbation, and external disturbances, although it is still a promising solution to the fuel cell control issues. Future exploration is highly expected on the multivariable adaptive control of fuel cell output voltage tracking, power regulation, temperature, humidity, efficiency, fuel and air flow rates, etc. The implementation of an adaptive control strategy is also worthy of development both for stationary and transportation systems, as well as for low and high power applications.

\section{References}

[1] J.T. Pukrushpan, A.G. Stefannopoulou, H. Peng, IEEE Control Syst. Mag. (2004) 30-46.

[2] M. Ceraolo, C. Miulli, A. Pozio, J. Power Sources 113 (2003) 131144.

[3] J. Golbert, D.R. Lewin, J. Power Sources 135 (2004) 135-151.

[4] P.R. Pathapati, X. Xue, J. Tang, Renewable Energy 30 (2005) 1-22.

[5] A. Paradkar, A. Davari, A. Feliachi, T. Biswas, J. Power Sources 128 (2004) $218-230$

[6] J.O. Schumacher, P. Gemmar, M. Denne, M. Zedda, M. Stueder, J. Power Sources 129 (2004) 143-151.

[7] F. Jurado, J.R. Saenz, IEEE Trans. Energy Convers. 18 (2) (2003) 342-347.

[8] R.T. Meyer, S. Revankar, A survey of PEM fuel cell system control models and control developments, Proceedings of FuelCell06: The Fourth International Conference on Fuel Cell Science, Engineering and Technology, Irvine, California, June 19-21, 2006. Paper No. 97030.

[9] R.F. Mann, J.C. Amphlett, M.A.I. Hooper, H.M. Jensen, B.A. Peppley, P.R. Roberge, J. Power Sources 86 (2000) 173-180.

[10] S. Yerramalla, A. Davari, A. Feliachi, T. Biswas, J. Power Sources 124 (2003) 104-113. 
[11] J.M. Correa, F.A. Farret, L.N. Canha, An analysis of the dynamic performance of proton exchange membrane fuel cells using an electrochemical model, IECON'01, The 27th Annual Conference of the IEEE Industrial Electronics Society, 2001, pp. 141-146.

[12] G.F. Franklin, J.D. Powell, A. Emami-Naeini, Feedback Control of Dynamic Systems, fifth ed., Pearson Prentice Hall, 2006.
[13] G.C. Goodwin, K.S. Sin, Adaptive Filtering Prediction and Control, Prentice-Hall, Englewood Cliffs, NJ, 1984.

[14] J. Larminie, A. Dicks, Fuel Cell Systems Explained, second ed., John Wiley \& Sons, 2003.

[15] D. Thirumalai, R.E. White, J. Appl. Electrochem. 30 (2000) 551559. 